\title{
Q-spaces and the foundations of quantum mechanics
}

\author{
Graciela Domenech ${ }^{1 *}$ Federico Holik ${ }^{1}$ and Décio $\mathrm{Krause}^{2 \dagger}$
}

September 29, 2008

\author{
${ }^{1}$ Instituto de Astronomía y Física del Espacio (IAFE) \\ Casilla de Correo 67, Sucursal 28, 1428 Buenos Aires, Argentina \\ and \\ 2 Departamento de Filosofia - Universidade Federal de Santa Catarina \\ P.O.Box 476, 88040-900 Forianópolis, SC - Brazil
}

\begin{abstract}
Our aim in this paper is to take quite seriously Heinz Post's claim that the non-individuality and the indiscernibility of quantum objects should be introduced right at the start, and not made a posteriori by introducing symmetry conditions. Using a different mathematical framework, namely, quasi-set theory, we avoid working within a labeltensor-product-vector-space-formalism, to use Redhead and Teller's words, and get a more intuitive way of dealing with the formalism of quantum mechanics, although the underlying logic should be modified. We build a vector space with inner product, the Q-space, using the non-classical part of quasi-set theory, to deal with indistinguishable elements. Vectors in Q-space refer only to occupation numbers and permutation operators act as the identity operator on them, reflecting in the formalism the fact of unobservability of permutations. Thus, this paper can be regarded as a tentative to follow and enlarge Heinsenberg's suggestion that new phenomena require the formation of a new "closed" (that is, axiomatic) theory, coping also with the physical theory's underlying logic and mathematics.
\end{abstract}

Key words: quasi-sets, particle number, Fock space, quantum indistinguishability.

\footnotetext{
${ }^{*}$ Fellow of the Consejo Nacional de Investigaciones Científicas y Técnicas (CONICET) - Argentina

${ }^{\dagger}$ Fellow of the Conselho Nacional de Desenvolvimento Científico e Tecnológico (CNPq) - Brazil
} 


\title{
1 Introduction
}

\begin{abstract}
"The transition in science from previously investigated fields of experience to new ones will never consist simply of the application of already known laws to these new fields. On the contrary, a really new field of experience will always lead to the crystallization of a new system of scientific concepts and laws (...). The advance from the parts already completed to those newly discovered, or to be newly erected, demands each time an intellectual jump, which cannot be achieved through the simple development of already existing knowledge." W. Heisenberg [15, p. 25], quoted from [3]
\end{abstract}

In his paper "Individuality in physics" ([24]), Heinz Post claimed that "[p]articles are non-individual in modern theory (...) [and] non-individuality has to be introduced right at the start". Our aim in this paper is to take quite seriously Heinz Post's claim. Usually, the way of dealing with indiscernible objects within the scope of classical logic and mathematics is by restricting them to certain structures, such that the relations and functions of the structure are not sufficient to individuate them. Saying in other words, such structures are not rigid, in the sense that there are automorphisms other than the identity function. For instance, within the additive group of the integers $\mathcal{Z}=\langle\mathbb{Z},+\rangle$, there is no way of distinguishing between two integers $n$ and $-n$, for the function $f(x)=-x$ is an automorphism of the structure. But in standard mathematics, such as in that one that can be built in Zermelo-Fraenkel set theory with the axiom of foundation (ZF), which we can assume bases all physical theories and which we shall identify with "classical mathematics", any structure can be extended to a rigid structure, that is, to a structure whose only automorphism is the identity function. That means that, outside the group $\mathcal{Z}$, for instance in the rigid extended structure $\mathcal{Z}^{\prime}=\langle\mathbb{Z},+,<\rangle$, we of course can distinguish, say, between 3 and -3 (for $-3<3$ but not reciprocally). The fact that any structure (built in ZF) can be extended to a rigid structure makes the indiscernibility of the objects something quite artificial. That means that, although we can deal with certain objects as if they were indiscernible, from "outside" of these structures these objects are not indiscernible, for they can be individualized in the extended rigid structures. In particular, in the "whole ZF", that is, in the well-founded "structure" $\mathcal{V}=\langle V, \in\rangle$, where $V$ is the von Neumann well founded universe and $\in$ is the membership relation, every representable object is an individual, in the sense that it obeys the laws of identity of classical (first or higher order) logic. In other words, any object $a$ can be distinguished from any other object $b$, say by the fact that it (and it 
only) belongs to its singleton $\{a\}$ (which can be identified -in extensional contexts- with the property "being identical to $a$ ", namely, $P(x) \stackrel{\text { def }}{=} x=a$ ).

In quantum physics, which is of course standardly build within classical mathematics (thus encompassing classical logic as well), the strategy is quite similar to that one mentioned above. When a system composed of particles of the same kind is considered, we start by labeling the particles we are dealing with, ${ }^{1}$ say by naming them "1", "2" and so on, and then consider the relevant Hilbert spaces $\mathcal{H}_{1}, \mathcal{H}_{2}$, etc., for each particle and, for the join system, we take the tensorial product $\mathcal{H}=\bigotimes_{i \in I} \mathcal{H}_{i}$. The base vectors $\left|\alpha_{1}\right\rangle \otimes\left|\alpha_{2}\right\rangle \otimes \ldots$, or simply $\left|\alpha_{1} \alpha_{2} \ldots\right\rangle$ must be so that for any permutation operator $P_{i j}$, which intuitively speaking exchanges the labels (hence, the particles) $i$ and $j$, we have

$$
P_{i j}\left|\alpha_{1} \ldots \alpha_{i} \ldots \alpha_{j} \ldots\right\rangle= \pm\left|\alpha_{1} \ldots \alpha_{j} \ldots \alpha_{i} \ldots\right\rangle,
$$

that is, the state of indistinguishable particles is left invariant by permutations. The plus sign stands for bosons, and the minus sign for fermions. This labeled-tensor-product-Hilbert-space formalism (LTPHSF), as called by Redhead and Teller [25], [26], [32], requires that symmetry conditions of this kind are introduced: we start from individuals, say by choosing a vector basis $\left\{\left|\alpha_{i}\right\rangle\right\}$ for a suitable Hilbert space, which serves as a kind of label to the particle. There is no scape. In order to talk of objects, we need to refer to them in some way (Toraldo di Francia says that our languages -including those of science- are "objectual" [33]), and this perhaps is due to the deficiencies of the language employed, taken from classical physics, which, as said Schrödinger, "gets off on the wrong foot" by initially assigning particle labels and then permuting them before extracting combinations of appropriate symmetry [17]. It is a challenge to find a suitable language that enables us to speak of indiscernible objects without making such first hypothesis about their individuality. Really, once indiscernibility lies in the core of quantum assumptions, perhaps we need to agree with Yuri Manin when he says that "quantum mechanics has not its own language" ([22, p. $84]$ ), for (ideally) a suitable language would refer to quantum objects without identifying them as individuals (this of course poses difficulties also for

\footnotetext{
${ }^{1}$ The term "particle" would not be taken literally as denoting neither tinny entities like usual bodies, nor entities as described by classical physics (for the differences, see [10, chap. 6]). For our argumentation, it does not matter what sort of entities they "really" are, but only that, yet far from the naïve idea associated with this word, "the particle concept [of particle] was not given up. After the quantum revolution, physicists still speak of particles" [10, p. 209]. We shall take this quotation ever in mind in this paper. An analogous consideration holds for the term "system".
} 
the use of quantifiers in physics). The introduction of symmetry conditions, say by choosing symmetric and anti-symmetric functions (or vectors), are necessary devices in the formalism. In the above mentioned papers, Redhead and Teller point to various puzzles caused by such an assumption, and propose the use of the Fock space formalism instead. We shall turn to their claim later. Yet, "for all practical purposes" (in Bell's words), LTPSF works quite well, as present day physics exemplifies, but rigorously speaking, we see that there is an enormous gap, for we are considering individuals at the start, and them "make" them non-individuals by some mathematical trick, like by "forgetting" that they are individuals and considering only certain quantities of them in each situation (this strategy was called "the Weyl strategy" in [12], where there are further philosophical discussions). Thus, to pursue Post's claim seems to be relevant.

We guess that quasi-set theory provides a language for dealing with collections of indiscernible elements right from the start. In this theory, the notion of indistinguishability (or indiscernibility) is taken as a primitive notion, and a definite concept of identity is restricted so that there may exist objects that are indiscernible without turning to be identical (to be the same object). Thus, the theory is non-Leibnizian, for his principle of the identity of indiscernibles is not valid in general: entities can have all the same properties without turning to be identical. We sketch the quasiset theory $\mathfrak{Q}$ in the next section (for further details, see [12, Chap. 7]). In 2 we review general notions on quasi-set theory. In 3 , we use the non classical part of quasi-set theory to construct a vector spaces with inner product, which is adequate to deal with bosons and fermions. In section 4 , we use this space to formulate quantum mechanics of indistinguishable particles without appealing to intermediate indexations. Finally, we expose our conclusions in 5 .

\section{Quasi-set theory}

We recall here some notions of quasi-set theory that will play an important role in what follows (for further details, see [12, Chap. 7]). We shall not present all the postulates and definitions of the theory, but just revise the main ideas and results which interest us here. Intuitively speaking a quasiset is a collection of indistinguishable (but not identical) objects. This of course is not a strict "definition" of a quasi-set, acting more or less as Cantor's "definition" of a set as "any collection into a whole $M$ of definite and separate [that is, distinguishable] objects $m$ of our intuition or our thought" 
(see the discussion in $[12, \S 6.4]$ ), giving no more than an intuitive account of the concept.

The quasi-set theory $\mathfrak{Q}$ was conceived to handle collections of indistinguishable objects, and was motivated by some considerations taken from quantum physics, mainly in what respects Schrödinger's idea that the concept of identity would not be applied to elementary particles [30, pp. 17-18]. Of course the theory can be developed independently of any formulation of quantum mechanics, but here we shall have this motivation always in mind. Our way to deal with indistinguishability is by assuming that expressions like $x=y$ are not always well formed. We express that by saying that the concept of identity does not apply to the entities denoted by $x$ and $y$ when they "refer" to quantum objects. Due to the lack of sense in applying the concept of identity to certain elements, informally, a quasi-set (qset), that is, a collection involving such objects, may be such that its elements cannot be identified by names, counted, ordered, although there is a sense in saying that these collections have a cardinal (not defined by means of ordinals, as usual -but see below). But we aim at to keep standard mathematics intact, so the theory is developed in a way that ZFU (and hence ZF, perhaps with the axiom of choice, ZFC) is a subtheory of $\mathfrak{Q}$. In other words, the theory is constructed so that it extends standard Zermelo-Fraenkel with Urelemente (ZFU) set theory; thus standard sets (of ZFU) can be viewed as particular qsets (that is, there are qsets that have all the properties of the sets of $\mathrm{ZFU}$; the objects in $\mathfrak{Q}$ corresponding to the Urelemente of $\mathrm{ZFU}$ are termed $M$-atoms). These objects will be called $\mathfrak{Q}$-sets, or just sets when there will be no confusion. But quasi-set theory encompasses another kind of Urelemente, the $m$-atoms, to which the standard theory of identity does not apply (that is, expressions like $x=y$ are not well formed if either $x$ or $y$ denote $m$-atoms). Thus, we can say that $\mathfrak{Q}$-sets are qsets whose transitive closure (defined as usual -see below) does not contain $m$-atoms (in other words, they are "constructed" in the "classical" part of the theory -see Fig. $1)$.

When $\mathfrak{Q}$ is used in connection with quantum physics, these $m$-atoms are thought of as representing quantum objects (henceforth, q-objects), and not necessarily they are 'particles'; waves or perhaps even strings (and whatever 'objects' sharing the property of indistinguishability of pointlike elementary particles) can be also be values of the variables of $\mathfrak{Q}$ (see [10, Chap. 6] for an account on the various ways to understand the word "particle" in connection to quantum physics). The lack of the concept of identity for the $m$-atoms makes them non-individuals in a sense, and it is mainly (but not only) to deal with collections of $m$-atoms that the theory was conceived. So, $\mathfrak{Q}$ is a 


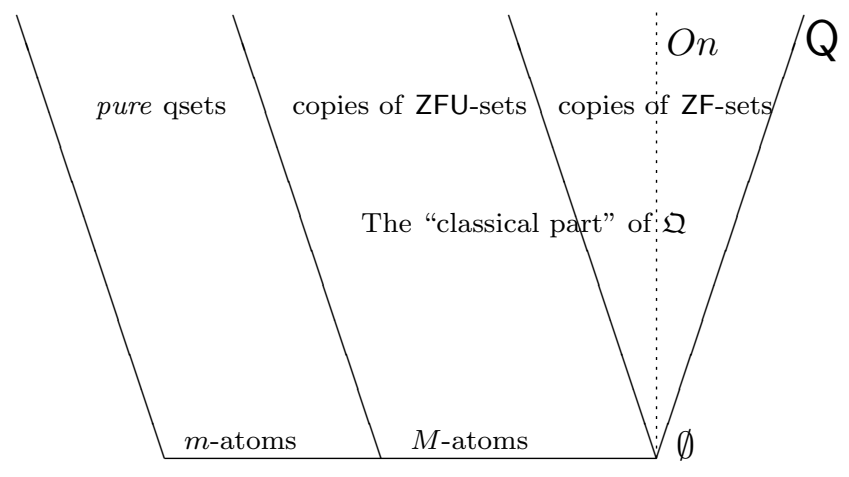

Figure 1: The quasi-set universe: $\mathbb{Q}$ is a "model" of $\mathfrak{Q}$.

theory of generalized collections of objects, involving non-individuals. For details about $\mathfrak{Q}$ and about its historical motivations, see [12, Chap. 7].

In order to distinguish between $\mathfrak{Q}$-sets and qsets that have $m$-atoms in their transitive closure, we write (in the metalanguage) $\{x: \varphi(x)\}$ for the former and $[x: \varphi(x)]$ for the latter. In $\mathfrak{Q}$, the so called 'pure' qsets have only q-objects as elements (although these elements may be not always indistinguishable from one another), and to them it is assumed that the usual notion of identity cannot be applied (that is, let us recall, $x=y$, so as its negation, $x \neq y$, are not a well formed formulas if either $x$ or $y$ stand for q-objects). Notwithstanding, there is a primitive relation $\equiv$ of indistinguishability having the properties of an equivalence relation, and a concept of extensional identity, not holding among $m$-atoms, is defined and has the properties of standard identity of classical set theories. More precisely, we write $x=_{E} y$ ( $x$ and $y$ are extensionally identical) iff they are both qsets having the same elements (that is, $\forall z(z \in x \leftrightarrow z \in y)$ ) or they are both $M$-atoms and belong to the same qsets (that is, $\forall z(x \in z \leftrightarrow y \in z)$ ). From now on, we shall use the symbol "=" for the extensional equality, except when explicitly mentioned.

Since the elements of a qset may have properties (and satisfy certain formulas), they can be regarded as indistinguishable without turning to be identical (that is, being the same object), that is, $x \equiv y$ does not entail $x=$ 
$y$. Since the relation of equality (and the concept of identity) does not apply to $m$-atoms, they can also be thought of as entities devoid of individuality. We remark further that if the 'property' $x=x$ (to be identical to itself, or self-identity, which can be defined for an object $a$ as $I_{a}(x) \stackrel{\text { def }}{=} x=a$ ) is included as one of the properties of the considered objects, then the so called Principle of the Identity of Indiscernibles (PII) in the form $\forall F(F(x) \leftrightarrow$ $F(y)) \rightarrow x=y$ is a theorem of classical second order logic, and hence there cannot be indiscernible but not identical entities (in particular, nonindividuals). Thus, if self-identity is linked to the concept of non-individual, and if quantum objects are to be considered as such, these entities fail to be self-identical, and a logical framework to accommodate them is in order (see [12] for further argumentation).

We have already discussed at length in the references given above (so as in other works) the motivations to build a quasi-set theory, and we shall not return to these points here, ${ }^{2}$ but before to continue we would like to make some few remarks on a common misunderstanding about PII and quantum physics. People generally think that spatio-temporal location is a sufficient condition for individuality. Thus, two electrons in different locations are discernible, hence distinct individuals. Leibniz himself prevented us about this claim (yet not directly about quantum objects of course), by saying that "it is not possible for two things to differ from one another in respect to place and time alone, but that is always necessary that there shall be some other internal difference" [20]. Leaving aside a possible interpretation for the word 'internal', we recall that even in quantum physics, fermions obey the Pauli Exclusion Principle, which says that two fermions (yes, they 'count' as more than one) cannot have all their quantum numbers (or 'properties') in common. Two electrons (which are fermions), one in the South Pole and another one in the North Pole, are not individuals in the standard sense (and we can do that without discussing the concepts of space and time). Here, by an individual we understand an object that obeys the classical theory of identity of classical (first or higher order) logic (extensional set theory included). In fact, we can say that the electron in the South Pole is described by the wave function $\psi_{S}(x)$, while the another one is described by $\psi_{N}(x)$ (words like 'another' in the preceding phrase are just ways of speech, done in the informal metalanguage). But the wave function of the joint system is given by $\psi_{S N}\left(x_{1}, x_{2}\right)=\psi_{S}\left(x_{1}\right) \psi_{N}\left(x_{2}\right)-\psi_{N}\left(x_{1}\right) \psi_{S}\left(x_{2}\right)$ (the function must be anti-symmetric in the case of fermions, that is, $\psi_{N S}\left(x_{1}, x_{2}\right)=$ $\left.-\psi_{N S}\left(x_{2}, x_{1}\right)\right)$, a superposition of the product wave functions $\psi_{S}\left(x_{1}\right) \psi_{N}\left(x_{2}\right)$

\footnotetext{
${ }^{2}$ But see [16], [4], [5], [17], [6], [11], [?], [19], [12].
} 
and $\psi_{S}\left(x_{2}\right) \psi_{N}\left(x_{1}\right)$. Such a superposition cannot be factorized. Furthermore, in the quantum formalism, the important thing is the square of the wave function, which gives the joint probability density; in the present case, we have $\left\|\psi_{S N}\left(x_{1}, x_{2}\right)\right\|^{2}=\left\|\psi_{S}\left(x_{1}\right) \psi_{N}\left(x_{2}\right)\right\|^{2}+\left\|\psi_{S}\left(x_{2}\right) \psi_{N}\left(x_{1}\right)\right\|^{2}-$ $2 \operatorname{Re}\left(\psi_{S}\left(x_{1}\right) \psi_{N}\left(x_{2}\right) \psi_{S}\left(x_{2}\right)^{*} \psi_{N}\left(x_{1}\right)^{*}\right)$. This last 'interference term' (though vanishing at large distances), cannot be dispensed with, and says that nothing, not even in mente Dei, can tell us which is the particular electron in the South Pole (and the same happens for the North Pole). As far as quantum physics is concerned, they really and truly have no identity in the standard sense (and hence they have not identity at all).

\subsection{The basic ideas of quasi-set theory}

Quasi-sets are the collections obtained by applying ZFU-like (Zermelo-Fraenkel plus Urelemente) axioms to a basic domain composed of $m$-atoms, $M$-atoms and aggregates of them. The theory still admits a primitive concept of quasicardinal which intuitively stands for the 'quantity' of objects in a collection. This is made so that certain quasi-sets $x$ (in particular, those whose elements are q-objects) may have a quasi-cardinal, written $q c(x)$, but not an ordinal. It is also possible to define a translation from the language of ZFU into the language of $\mathfrak{Q}$ in such a way so that there is a 'copy' of ZFU in $\mathfrak{Q}$ (the 'classical' part of $\mathfrak{Q}$ ). In this copy, all the usual mathematical concepts can be defined (inclusive the concept of ordinal for the $\mathfrak{Q}$-sets), and the $\mathfrak{Q}$-sets turn out to be those quasi-sets whose transitive closure (this concept is like the usual one) does not contain $m$-atoms. ${ }^{3}$

To understand the basic involved ideas, let us consider the three protons and the four neutrons in the nucleus of a ${ }^{7} \mathrm{Li}$ atom. As far as quantum mechanics goes, nothing distinguishes these three protons. If we regard these protons as forming a quasi-set, its quasi-cardinal should be 3 , and there is no apparent contradiction in saying that there are also 3 subquasi-sets with 2 elements each, despite we can't distinguish their elements, and so on. So, it is reasonable to postulate that the quasi-cardinal of the power quasi-set of $x$ is $2^{q c(x)}$. Whether we can distinguish among these subquasi-sets is a matter which does not concern logic.

\footnotetext{
${ }^{3}$ So, we can make sense to the primitive concept of quasi-cardinal of a quasi-set $x$ as being a cardinal defined in the 'classical' part of the theory. The reason to take the concept of quasi-cardinal as a primitive concept will appear below, when we make reference to the distinction between cardinals and ordinals. The first two authors of this paper have defined the quasi-cardinal for finite qsets; see [9]. Independently, Becker has extending this idea with other considerations [2].
} 
In other words, we may consistently (with the axiomatics of $\mathfrak{Q}$ ) reason as if there are three entities in our quasi-set $x$, but $x$ must be regarded as a collection for which it is not possible to discern its elements as individuals. The theory does not enable us to form the corresponding singletons. The grounds for such kind of reasoning has been delineated by Dalla Chiara and Toraldo di Francia as partly theoretical and partly experimental. Speaking of electrons instead of protons, they note that in the case of the helium atom we can say that there are two electrons because, theoretically, the appropriate wave function depends on six coordinates and thus "we can therefore say that the wave function has the same degrees of freedom as a system of two classical particles". ${ }^{4}$ Dalla Chiara and Toraldo di Francia have also noted that, "[e]xperimentally, we can ionize the atom (by bombardment or other means) and extract two separate electrons ..." (ibid.).

Of course, the electrons can be counted as two only at the moment of measurement; as soon as they interact with other electrons (in the measurement apparatus, for example) they enter into entangled states once more. It is on this basis that one can assert that there are two electrons in the helium atom or six in the $2 p$ level of the sodium atom or (by similar considerations) three protons in the nucleus of a ${ }^{7} \mathrm{Li}$ atom (and it may be contended that the 'theoretical' ground for reasoning in this way also depends on these experimental considerations, together with the legacy of classical metaphysics). On this basis it is stated the axiom of 'weak extensionality' of $\mathfrak{Q}$, which says that those quasi-sets that have the same quantity of elements of the same sort (in the sense that they belong to the same equivalence class of indistinguishable objects) are indistinguishable.

This axiom has interesting consequences. As we have said, there is no space here for the details, but let us mention just one of them which is related to the above discussion on the non observability of permutations in quantum physics, which is one of the most basic facts regarding indistinguishable quanta. In standard set theories, if $w \in x$, then of course $(x-\{w\}) \cup\{z\}=x$ iff $z=w$. That is, we can 'exchange' (without modifying the original arrangement) two elements iff they are the same elements, by force of the axiom of extensionality. But in $\mathfrak{Q}$ we can prove the following theorem, where $z^{\prime}$ (and similarly $w^{\prime}$ ) stand for a quasi-set with quasi-cardinal 1 whose only element is indistinguishable from $z$ (respectively, from $w$-the reader shouldn't think that this element is identical to either $z$ or $w$, for the

\footnotetext{
${ }^{4}$ Op. cit., p. 268. This might be associated to the legacy of Schrödinger, who says that this kind of formulation "gets off on the wrong foot" by initially assigning particle labels and then permuting them before extracting combinations of appropriate symmetry [31].
} 
relation of equality doesn't apply here; the set theoretical operations can be understood according to their usual definitions):

[Unobservability of Permutations] Let $x$ be a finite quasi-set such that $x$ does not contain all indistinguishable from $z$, where $z$ is an $m$-atom such that $z \in x$. If $w \equiv z$ and $w \notin x$, then there exists $w^{\prime}$ such that

$$
\left(x-z^{\prime}\right) \cup w^{\prime} \equiv x
$$

Supposing that $x$ has $n$ elements, then if we 'exchange' their elements $z$ by correspondent indistinguishable elements $w$ (set theoretically, this means performing the operation $\left.\left(x-z^{\prime}\right) \cup w^{\prime}\right)$, then the resulting quasi-set remains indistinguishable from the original one. In a certain sense, it is not important whether we are dealing with $x$ or with $\left(x-z^{\prime}\right) \cup w^{\prime}$. This of course gives a 'set-theoretical' sense to the following claim made by Roger Penrose:

"[a]ccording to quantum mechanics, any two electrons must necessarily be completely identical [in the physicist's jargon, that is, indistinguishable], and the same holds for any two protons and for any two particles whatever, of any particular kind. This is not merely to say that there is no way of telling the particles apart; the statement is considerably stronger than that. If an electron in a person's brain were to be exchanged with an electron in a brick, then the state of the system would be exactly the same state as it was before, not merely indistinguishable from it! The same holds for protons and for any other kind of particle, and for the whole atoms, molecules, etc. If the entire material content of a person were to be exchanged with the corresponding particles in the bricks of his house then, in a strong sense, nothing would be happened whatsoever. What distinguishes the person from his house is the pattern of how his constituents are arranged, not the individuality of the constituents themselves" [28, p. 32].

Within $\mathfrak{Q}$ we can express that 'permutations are not observable', without necessarily introducing symmetry postulates, and in particular to derive 'in a natural way' the quantum statistics (see [18], [12, Chap. 7]).

\section{The Q-space}

In the standard formulation of quantum mechanics, pure states of quantum systems are represented by normalized to unit vectors in a Hilbert space. 
In the case of identical particles, the vectors representing their states are symmetrized or antisymmetrized, as mentioned above. In this section, we will use $\mathfrak{Q}$ to construct a vector space, which we will call $\mathfrak{Q}$-space, in which the states are defined without labeling particles for they are represented by $m$-atoms. The structure of this space will result analogous to that of the Fock-space.

\subsection{Motivation}

Let us analyze with a deeper detail how quantum mechanics deals with a system of two indistinguishable particles, just to introduce some notation and to motivate our construction. Recall that the usual construction of a vector space -and of the whole formalism of quantum mechanics- makes use of set theory, which presupposes the individuality and distinguishability of the elements of any set. First the Hilbert space $\mathcal{H}=\mathcal{H}_{1} \otimes \mathcal{H}_{2}$ is constructed up from the one particle spaces $\mathcal{H}_{1}$ and $\mathcal{H}_{2}$. We use Dirac notation for simplicity. Let $\{|\alpha\rangle\}$ be a basis set of $\mathcal{H}_{i}$. Then, $\{|\alpha\rangle \otimes|\beta\rangle\}$ is a basis for $\mathcal{H}$. $\alpha$ and $\beta$ run over all possible values of the corresponding physical magnitudes and it is understood that the first ket corresponds to the particle labeled "1" and the second to the one labeled "2".

The scalar product of any two basis vectors is given by:

$$
\left(\left\langle\alpha|\otimes\langle\beta|)\left(\left|\alpha^{\prime}\right\rangle \otimes\left|\beta^{\prime}\right\rangle\right)=\left\langle\alpha \mid \alpha^{\prime}\right\rangle\left\langle\beta \mid \beta^{\prime}\right\rangle\right.\right.
$$

and, in general, the scalar product between two product vectors $|\psi\rangle \otimes|\varphi\rangle$ and $\left|\psi^{\prime}\right\rangle \otimes\left|\varphi^{\prime}\right\rangle$ of the product space is given by:

$$
\left(\left\langle\psi|\otimes\langle\varphi|)\left(\left|\psi^{\prime}\right\rangle \otimes\left|\varphi^{\prime}\right\rangle\right)=\left\langle\psi \mid \psi^{\prime}\right\rangle\left\langle\varphi \mid \varphi^{\prime}\right\rangle .\right.\right.
$$

It is worth noting that when $\alpha$ and $\beta$ are different, $|\alpha\rangle \otimes|\beta\rangle$ will be not the same vector as $|\beta\rangle \otimes|\alpha\rangle$. Thus, in general, if $\left|\psi^{\prime}\right\rangle$ and $\left|\varphi^{\prime}\right\rangle$ are linear combinations of basis vectors, it results that:

$$
\left(\left\langle\psi|\otimes\langle\varphi|)\left(\left|\psi^{\prime}\right\rangle \otimes\left|\varphi^{\prime}\right\rangle\right) \neq\left(\left\langle\psi|\otimes\langle\varphi|)\left(\left|\varphi^{\prime}\right\rangle \otimes\left|\psi^{\prime}\right\rangle\right)\right.\right.\right.\right.
$$

and this has no sense when dealing with indistinguishable particles. To solve this difficult, the symmetrization postulate is assumed.

Our aim is to develop a procedure that takes into account indistinguishability from the start, so we recall in which steps artificial labeling has occurred. First of all, one assigns vectors states to each particle in their corresponding Hilbert spaces and "names" in some way these spaces to perform the tensor product. Informally, one says that one is making the product of 
the Hilbert space of particle "1" and the Hilbert space of particle "2", and does the same thing for the resulting states. As one arrives at a situation in which particles seem to be distinguishable, one applies a symmetrization postulate. Then, when defining the scalar product, the differentiation of state spaces is maintained when taking brackets, the bra of particle "1" with the ket of particle "1" and the same for particle "2". Thus, there are two steps to be avoided: the use of the tensor product and this differentiation in the scalar product.

To introduce the formal construction which will be developed in the next sections, consider the possibility of a definition of a scalar product resembling the following: Let $\{|\alpha\rangle \otimes|\beta\rangle\}$ and $\left\{\left|\alpha^{\prime}\right\rangle \otimes\left|\beta^{\prime}\right\rangle\right\}$ be two basis vectors of the state space of the two particle system, then we could define their scalar product as

$$
\left(\left\langle\alpha|\otimes\langle\beta|) \circ\left(\left|\alpha^{\prime}\right\rangle \otimes\left|\beta^{\prime}\right\rangle\right)=\delta_{\alpha \alpha^{\prime}} \delta_{\beta \beta^{\prime}}+\delta_{\alpha \beta^{\prime}} \delta_{\beta \alpha^{\prime}}\right.\right.
$$

For any two vectors $|\psi\rangle \otimes|\varphi\rangle$ and $\left|\psi^{\prime}\right\rangle \otimes\left|\varphi^{\prime}\right\rangle$ that are linear combinations of the basis ones, one would obtain:

$$
\left(\left\langle\psi|\otimes\langle\varphi|) \circ\left(\left|\psi^{\prime}\right\rangle \otimes\left|\varphi^{\prime}\right\rangle\right)=\left\langle\psi \mid \psi^{\prime}\right\rangle\left\langle\varphi \mid \varphi^{\prime}\right\rangle+\left\langle\psi \mid \varphi^{\prime}\right\rangle\left\langle\varphi \mid \psi^{\prime}\right\rangle .\right.\right.
$$

It is easy to verify that this product satisfies:

$$
\left(\left\langle\psi|\otimes\langle\varphi|) \circ(|\psi\rangle \otimes|\varphi\rangle)=|\psi|^{2}|\varphi|^{2}+|\langle\psi \mid \varphi\rangle|^{2} \geq 0,\right.\right.
$$

and also:

$$
\left(\left\langle\psi^{\prime}\left|\otimes\left\langle\varphi^{\prime}\right|\right) \circ(|\psi\rangle \otimes|\varphi\rangle)=\left(\left(\left\langle\psi|\otimes\langle\varphi|) \circ\left(\left|\psi^{\prime}\right\rangle \otimes\left|\varphi^{\prime}\right\rangle\right)\right)^{*} .\right.\right.\right.\right.
$$

where ${ }^{*}$ stands for complex conjugation. Another possibility to be considered is

$$
\left(\left\langle\psi|\otimes\langle\varphi|) \bullet\left(\left|\psi^{\prime}\right\rangle \otimes\left|\varphi^{\prime}\right\rangle\right)=\left\langle\psi \mid \psi^{\prime}\right\rangle\left\langle\varphi \mid \varphi^{\prime}\right\rangle-\left\langle\psi \mid \varphi^{\prime}\right\rangle\left\langle\varphi \mid \psi^{\prime}\right\rangle\right.\right.
$$

This "product" clearly depends on the order of the terms, and it is defined up to a minus sign. But recall that in quantum mechanics we are interested in squared probability amplitudes and its square does not depend on the order. Furthermore, the product $\bullet$ has the following interesting property:

$$
(\langle\psi|\otimes\langle\psi|) \bullet(|\psi\rangle \otimes|\psi\rangle)=\langle\psi \mid \psi\rangle\langle\psi \mid \psi\rangle-\langle\psi \mid \psi\rangle\langle\psi \mid \psi\rangle=0
$$

and this will turn of great importance, because if we interpret $|\psi\rangle \otimes|\psi\rangle$ as an state with two fermions in the same state, the state is a vector of null 
norm, and thus, null probability, and also its scalar product with any other vector is zero:

$$
(\langle\varphi|\otimes\langle\phi|) \bullet(|\psi\rangle \otimes|\psi\rangle)=\langle\phi \mid \psi\rangle\langle\varphi \mid \psi\rangle-\langle\phi \mid \psi\rangle\langle\varphi \mid \psi\rangle=0
$$

Moreover, using Cauchy-Schwartz inequality we have that

$$
\left(\left\langle\psi|\otimes\langle\varphi|) \bullet(|\psi\rangle \otimes|\varphi\rangle)=|\psi|^{2}|\varphi|^{2}-|\langle\psi \mid \varphi\rangle|^{2} \geq 0,\right.\right.
$$

or

$$
\left(\left\langle\psi|\otimes\langle\varphi|) \bullet(|\psi\rangle \otimes|\varphi\rangle)=-|\psi|^{2}|\varphi|^{2}+|\langle\psi \mid \varphi\rangle|^{2} \leq 0 .\right.\right.
$$

These two possibilities come from the ambiguity in the sign when we define "•". This ambiguity will be solved later.

\subsection{Construction of the $\mathfrak{Q}$-space}

In the following we apply the guiding ideas discussed above to define a product in a $\mathfrak{Q}$-space constructed using the quasi-set theory $\mathfrak{Q}$.

\subsubsection{Quasi-functions}

Let us consider a $\mathfrak{Q}$-set $\epsilon={ }_{E}\left\{\epsilon_{i}\right\}_{i \in I}$, where $I$ is an arbitrary (denumerable or not) collection of indexes, such that $Z(\epsilon)$. From now own, by a "set" we mean a $\mathfrak{Q}$-set, and "=" stands for " $={ }_{E}$ ", except if explicitly mentioned. We also recall that all the usual mathematical concepts mentioned below can be obtained in the "classical" part of $\mathfrak{Q}$. We take the elements $\epsilon_{i}$ to represent the eigenvalues of a physical magnitude of interest. To fix the ideas, they may be the energy eigenvalues of the Hamiltonian $H$ of the system, $H\left|\varphi_{i}\right\rangle=\epsilon_{i}\left|\varphi_{i}\right\rangle$, with $\left|\varphi_{i}\right\rangle$ being the corresponding eigenstates. The construction we present is, of course, independent of this particular choice. Consider then the quasi-functions $f, f: \epsilon \longrightarrow \mathcal{F}_{p}$, where $\mathcal{F}_{p}$ is the quasi-set formed of all finite and pure quasi-sets. $f$ is the quasi-set formed of ordered pairs $\left\langle\epsilon_{i} ; x\right\rangle$ with $\epsilon_{i} \in \epsilon$ and $x \in \mathcal{F}_{p}$. Let us choice these quasi-functions in such a way that whenever $\left\langle\epsilon_{i_{k}} ; x\right\rangle$ and $\left\langle\epsilon_{i_{k^{\prime}}} ; y\right\rangle$ belong to $f$ and $k \neq k^{\prime}$, then $x \cap y=\emptyset$. Let us further assume that the sum of the quasi-cardinals of the quasi-sets which appear in the image of each of these quasi-functions is finite, and then, $q c(x)=0$ for every $x$ in the image of $f$, except for a finite number of elements of $\epsilon$. Let us call $\mathcal{F}$ the quasi-set formed of these quasi-functions. If $\left\langle x ; \epsilon_{i}\right\rangle$ is a pair of $f \in \mathcal{F}$, we will interpret that the energy level $\epsilon_{i}$ has occupation number $q c(x)$. These quasi-functions will be represented by symbols such as $f_{\epsilon_{i_{1}} \epsilon_{i_{2}} \ldots \epsilon_{i_{m}}}$ (or by the same symbol with 
permuted indexes). This indicates that the levels $\epsilon_{i_{1}} \epsilon_{i_{2}} \ldots \epsilon_{i_{m}}$ are occupied. It will be taken as convention that if the symbol $\epsilon_{i_{k}}$ appears $j$-times, then the level $\epsilon_{i_{k}}$ has occupation number $j$. For example, the symbol $f_{\epsilon_{1} \epsilon_{1} \epsilon_{1} \epsilon_{2} \epsilon_{3}}$ means that the level $\epsilon_{1}$ has occupation number 3 while the levels $\epsilon_{2}$ and $\epsilon_{3}$ have occupation numbers 1 . The levels that do not appear have occupation number zero.

These quasi-functions will be used to construct quantum states. It is worth to say that, because of the utilization of pure quasi-sets with indistinguishable elements, there is no reference to particle indexation. The only reference is to the occupation numbers, because permutations make no sense here, as it should be. Let us consider, for example, the quasi-function $f_{\epsilon_{1} \epsilon_{1} \epsilon_{1} \epsilon_{2} \epsilon_{3}}$. As we have said above, we interpret this as a state in which the level 1 has occupation number three, the levels 2 and 3 only one, and the others zero. Thus, a permutation of particles makes no change because the quasi-function $f_{\epsilon_{1} \epsilon_{1} \epsilon_{1} \epsilon_{2} \epsilon_{3}}$ is a collection of ordered pairs. These pairs are $\left\langle\epsilon_{1} ; x\right\rangle,\left\langle\epsilon_{2} ; y\right\rangle,\left\langle\epsilon_{3} ; z\right\rangle$ and $\left\langle\epsilon_{n} ; \emptyset\right\rangle$ (for $n>3$ ), where $x, y$ and $z$ are pure and disjoint quasi-sets which satisfy $q c(x)=3$ and $q c(y)=1=q c(z)$. Thus, permutation of two particles is formally represented by the procedure that takes an element of, say, $x$ and interchanges it with an element of $y$ (or $z$ ). But it is a theorem of $\mathfrak{Q}$ that permutation of $m$-atoms gives place to indistinguishable quasi-sets (unobservability of permutations). By definition, we have $\left\langle\epsilon_{1} ; x\right\rangle=\left[\left[\epsilon_{1}\right] ;\left[\epsilon_{1} ; x\right]\right]$. Also by definition, $\left[\epsilon_{1} ; x\right]$ is the collection of all the indistinguishable from either $\epsilon_{1}$ or $x$ (taken from some previously given qset). For this reason, if we replace $x$ by $x^{\prime}$, with $x \equiv x^{\prime}$ we will obtain $\left[\epsilon_{1} ; x\right]=\left[\epsilon_{1} ; x^{\prime}\right]$. Thus, we obtain $\left\langle\epsilon_{1} ; x\right\rangle=\left\langle\epsilon_{1} ; x^{\prime}\right\rangle$ and the ordered pairs of the 'permuted' quasi-function will be the same and, consequently, the new quasi-function is again $f_{\epsilon_{1} \epsilon_{1} \epsilon_{1} \epsilon_{2} \epsilon_{3}}$. We thus see that the permutation of indistinguishable elements does not produce changes in the quasi-functions and, then, in any vector space constructed using them, the permutation operation will be reduced to identity.

It is important to point out that the order of the indexes in a quasifunction $f_{\epsilon_{i_{1}} \epsilon_{i_{2}} \ldots \epsilon_{i_{n}}}$ has no meaning at all because up to now, there is no need to define any particular order in $\epsilon$, the domain of the quasi-functions of $\mathcal{F}$. Nevertheless, we may define an order in the following way. For each quasi-function $f \in \mathcal{F}$, let $\left\{\epsilon_{i_{1}} \epsilon_{i_{2}} \ldots \epsilon_{i_{m}}\right\}$ be the quasi-set formed by the elements of $\epsilon$ such that $\left\langle\epsilon_{i_{k}}, X\right\rangle \in f$ and $q c(X) \neq 0(k=1 \ldots m)$. We call $\operatorname{supp}(f)$ this quasi-set (the support of $f$ ). Then consider the pair $\langle o, f\rangle$, where $o$ is a bijective quasi-function:

$$
o:\left\{\epsilon_{i_{1}} \epsilon_{i_{2}} \ldots \epsilon_{i_{m}}\right\} \longrightarrow\{1,2, \ldots, m\}
$$


Each of these quasi-functions $o$ define an order on $\operatorname{supp}(f)$. For each $f \in \mathcal{F}$, if $q c(\operatorname{supp}(f))=m$, then, there are $m$ ! orderings. Then, let $\mathcal{O F}$ be the quasi-set formed by all the pairs $\langle o, f\rangle$, where $f \in \mathcal{F}$ and $o$ is a a particular ordering in $\operatorname{supp}(f)$. Thus, $\mathcal{O F}$ is the quasi-set formed by all the quasifunctions of $\mathcal{F}$ with ordered support. For this reason, if we now say that $f_{\epsilon_{i_{1}} \epsilon_{i_{2}} \ldots \epsilon_{i_{n}}} \in \mathcal{O} \mathcal{F}$, we will be speaking of a quasifunction $f \in \mathcal{F}$ and of an special ordering of $\left\{\epsilon_{i_{1}} \epsilon_{i_{2}} \ldots \epsilon_{i_{n}}\right\}$. For the sake of simplicity, we will use the same notation as before. But now the order of the indexes is meaningful. It is also important to remark, that the order on the indexes must not be understood as a labeling of particles, for it easy to check that, as above, the permutation of particles does not give place to a new element of $\mathcal{O F}$. This is so because a permutation of particles operating on a pair $\langle o, f\rangle \in \mathcal{O F}$ will not change $f$, and so, will not alter the ordering. We will use the elements of $\mathcal{O} \mathcal{F}$ later, when we deal with fermions.

\subsubsection{Vector space structure}

A linear space structure is required to adequately represent quantum states. To equip $\mathcal{F}$ and $\mathcal{O} \mathcal{F}$ with such a structure, we need to define two operations " $\star$ " and "+", a product by scalars and an addition of their elements, respectively. We will construct a vector space starting from the quasi-functions of the quasi-sets $\mathcal{F}$ (or equivalently $\mathcal{O} \mathcal{F}$ ) defined above. Call $C$ the collection of quasi-functions which assign to every $f \in \mathcal{F}$ (or $f \in \mathcal{O} \mathcal{F}$ ) a complex number. That is, a quasi-function $c \in C$ is a collection of ordered pairs $\langle f ; \lambda\rangle$, where $f \in \mathcal{F}$ (or $f \in \mathcal{O F}$ ) and $\lambda$ a complex number. Let $C_{0}$ be the subset of $C$ such that, if $c \in C_{0}$, then $c(f)=0$ for almost every $f \in \mathcal{O} \mathcal{F}$ (i.e., $c(f)=0$ for every $f \in \mathcal{O} \mathcal{F}$ except for a finite number of quasi-functions). We can define in $C_{0}$ a sum and a product by scalars in the same way as it is usually done with functions as follows.

Definition 3.1 Let $\alpha, \beta$ and $\gamma \in \mathcal{C}$, and $c, c_{1}$ and $c_{2}$ be quasi-functions of $C_{0}$, then

$$
\begin{gathered}
(\gamma * c)(f) \stackrel{\text { def }}{=} \gamma(c(f)) \\
\left(c_{1}+c_{2}\right)(f) \stackrel{\text { def }}{=} c_{1}(f)+c_{2}(f)
\end{gathered}
$$

The quasi-function $c_{0} \in C_{0}$ such that $c_{0}(f)=0$, for any $f \in F$, acts as the null element of the sum, for

$$
\left(c_{0}+c\right)(f)=c_{0}(f)+c(f)=0+c(f)=c(f), \forall f .
$$


With the sum and the multiplication by scalars defined above we have that $\left(C_{0},+, *\right)$ is a complex vector space. Each one of the quasi-functions of $C_{0}$ should be interpreted in the following way. If $c \in C_{0}$ (and $c \neq c_{0}$ ), let $f_{1}, f_{2}, f_{3}, \ldots, f_{n}$ be the only functions of $C_{0}$ which satisfy $c\left(f_{i}\right) \neq 0(i=$ $1, \ldots, n)$. These quasi-functions exist because, as we have said above, the quasi-functions of $C_{0}$ are zero except for a finite number of quasi-functions of $\mathcal{F}$. If $\lambda_{i}$ are complex numbers which satisfy that $c\left(f_{i}\right)=\lambda_{i}(i=1, \ldots, n)$, we will make the association

$$
c \approx\left(\lambda_{1} f_{1}+\lambda_{2} f_{2}+\cdots+\lambda_{n} f_{n}\right) .
$$

The symbol $\approx$ must be understood in the sense that we use this notation to represent the quasi-function $c$. The idea is that the quasi-function $c$ represents the pure state which is a linear combination of the states represented by the quasi-functions $f_{i}$ according to the interpretation given above. As a particular case of this notation, we have that if $c_{j} \in C_{0}$ are the quasifunctions such that $c_{j}\left(f_{i}\right)=\delta_{i j}$ ( $\delta_{i j}$ is the Kronecker symbol), then $c_{j} \approx f_{j}$ and in a similar way $\lambda * c_{j} \approx \lambda f_{j}$. In this space, the vectors $c_{j}$ are the "natural" basis vectors, while the others are linear combinations of them.

\subsubsection{Scalar products}

With the aid of a vector space structure, we can express quantum superpositions. In order to calculate probabilities and mean values, we need to introduce the notion of scalar product. In the following, we will introduce two different products for bosons and fermions separately, following the ideas of Section 3.1. Let us do it first for bosons.

Definition 3.2 Let $\delta_{i j}$ be the Kronecker symbol and $f_{\epsilon_{i_{1}} \epsilon_{i_{2}} \ldots \epsilon_{i_{n}}}$ and $f_{\epsilon_{i_{1}^{\prime}} \epsilon_{i_{2}^{\prime}} \ldots \epsilon_{i_{m}^{\prime}}}$ two basis vectors, then

$$
f_{\epsilon_{i_{1}} \epsilon_{i_{2}} \ldots \epsilon_{i_{n}}} \circ f_{\epsilon_{i_{1}^{\prime}} \epsilon_{i_{2}^{\prime}} \ldots \epsilon_{i_{m}^{\prime}}} \stackrel{\text { def }}{=} \delta_{n m} \sum_{p} \delta_{i_{1} p i_{1}^{\prime}} \delta_{i_{2} p i_{2}^{\prime}} \ldots \delta_{i_{n} p i_{n}^{\prime}}
$$

The sum is extended over all the permutations of the index set $i^{\prime}=\left(i_{1}^{\prime}, i_{2}^{\prime}, \ldots, i_{n}^{\prime}\right)$ and for each permutation $p, p i^{\prime}=\left(p i_{1}^{\prime}, p i_{2}^{\prime}, \ldots, p i_{n}^{\prime}\right)$.

This product can be easily extended over linear combinations:

$$
\left(\sum_{k} \alpha_{k} f_{k}\right) \circ\left(\sum_{k} \alpha_{k}^{\prime} f_{k}^{\prime}\right) \stackrel{\text { def }}{=} \sum_{k j} \alpha_{k}^{*} \alpha_{j}^{\prime}\left(f_{k} \circ f_{j}^{\prime}\right)
$$

On the other hand, we can consider another "•" product as follows, which will be adequate for fermions: 
Definition 3.3 Let $\delta_{i j}$ be the Kronecker symbol and $f_{\epsilon_{i_{1}} \epsilon_{i_{2}} \ldots \epsilon_{i_{n}}}$ and $f_{\epsilon_{i_{1}^{\prime}} \epsilon_{i_{2}^{\prime}} \ldots \epsilon_{i_{m}^{\prime}}}$ two basis vectors, then

$$
f_{\epsilon_{i_{1}} \epsilon_{i_{2}} \ldots \epsilon_{i_{n}}} \bullet f_{\epsilon_{i_{1}^{\prime}} \epsilon_{i_{2}^{\prime}} \ldots \epsilon_{i_{m}^{\prime}}} \stackrel{\text { def }}{=} \delta_{n m} \sum_{p} \sigma_{p} \delta_{i_{1} p i_{1}^{\prime}} \delta_{i_{2} p i_{2}^{\prime}} \ldots \delta_{i_{n} p i_{n}^{\prime}}
$$

where:

$$
\sigma_{p}= \begin{cases}1, & \text { if } p \text { is even } \\ -1, & \text { if } p \text { is odd }\end{cases}
$$

The result of this product is an antisymmetric sum of the indexes which appear in the quasi-functions. In order that the product is well defined, the quasi-functions must belong to $\mathcal{O F}$. Once this product is defined over the basis functions, we can extend it to linear combinations, in a similar way as in (16). If the occupation number of a product is more or equal than two, then the vector has null norm. As it is a vector of null norm, the product of this vector with any other vector of the space would yield zero, and thus the probability of observing a system in a state like it vanishes. This means that we can add to any physical state an arbitrary linear combination of null norm vectors for they do not contribute to the scalar product, which is the meaningful quantity.

We have defined two products, "o" and "•", that are adequate for bosons and fermions, respectively. We will return to this point in the following section.

We point out that to formulate quantum mechanics in such a way that no reference to particle individuality is made, we need to avoid labeling in state vectors as much as in operators representing observable quantities. As it is known, in the Fock-space formalism, the observables can be written in terms of creation and annihilation operators, avoiding particle labeling. In the following section we will introduce creation and annihilation operators in order to express observable quantities, without making appeal to particle labeling in the operators themselves.

\section{The construction of quantum mechanics using Q-spaces}

In this section we will first briefly review for completeness the formulation of quantum mechanics using the Fock-space formalism. After that, we will rewrite that formulation using the language of the $\mathfrak{Q}$-space. 


\subsection{Fock-space formalism}

As is well known, the standard formulation of quantum mechanics and the Fock-space formulation are deeply connected. Equivalence with wave mechanics is studied (for example) in [27]. Here we briefly recall some basic notions of the standard formalism to fix notation. We call $T_{1}$ the kinetic energy of a single particle and $V_{1}$ the external potential acting on it. For $n$ particles we have:

$$
T_{n} \stackrel{\text { def }}{=} \sum_{i=1}^{n} T_{1}\left(r_{i}\right)
$$

and the same for the external potential. We represent by

$$
V_{n} \stackrel{\text { def }}{=} \sum_{i>j=1}^{n} V_{2}\left(\mathbf{r}_{i}, \mathbf{r}_{j}\right)
$$

the pairwise interaction potential. Thus, the total hamiltonian operator is given by

$$
H_{n}=\sum_{i=1}^{n}\left[T_{1}\left(\mathbf{r}_{i}\right)+V_{1}\left(\mathbf{r}_{i}\right)+\sum_{i>j=1}^{n} V_{2}\left(\mathbf{r}_{i}, \mathbf{r}_{j}\right)\right]
$$

The $n$-particles wave function is written as

$$
\Psi_{n}\left(\mathbf{r}_{1}, \ldots, \mathbf{r}_{n}\right)
$$

The standard Fock-space is built up from the one particle Hilbert spaces. Let $\mathcal{H}$ be a Hilbert space and define:

$$
\begin{aligned}
\mathcal{H}^{0} & =\mathcal{C} \\
\mathcal{H}^{1} & =\mathcal{H} \\
\mathcal{H}^{2} & =\mathcal{H} \otimes \mathcal{H} \\
\vdots & \\
\mathcal{H}^{n} & =\mathcal{H} \otimes \cdots \otimes \mathcal{H}
\end{aligned}
$$

If no symmetry condition is required for the states, the Fock-space is constructed as the direct sum of $n$ particles Hilbert spaces:

$$
\mathcal{F}=\bigoplus_{n=0}^{\infty} \mathcal{H}^{n}
$$


When dealing with bosons or fermions, the standard procedure to obtain the physical space state is as follows. Given a vector $v=v_{1} \otimes \cdots \otimes v_{n} \in \mathcal{H}^{n}$, define:

$$
\sigma^{n}(v)=\left(\frac{1}{n !}\right) \sum_{P} P\left(v_{1} \otimes \cdots \otimes v_{n}\right)
$$

and:

$$
\tau^{n}(v)=\left(\frac{1}{n !}\right) \sum_{P} s^{p} P\left(v_{1} \otimes \cdots \otimes v_{n}\right)
$$

where:

$$
s^{p}= \begin{cases}1, & \text { if } \mathrm{p} \text { is even } \\ -1, & \text { if } \mathrm{p} \text { is odd }\end{cases}
$$

It is important to realize that in this construction, particles are labeled and then symmetry conditions are imposed by state symmetrization. Then, calling

$$
\mathcal{H}_{\sigma}^{n}=\left\{\sigma^{n}(v): v \in \mathcal{H}^{n}\right\}
$$

and:

$$
\mathcal{H}_{\tau}^{n}=\left\{\tau^{n}(v): v \in \mathcal{H}^{n}\right\}
$$

we have the Fock-space

$$
\mathcal{F}_{\sigma}=\bigoplus_{n=0}^{\infty} \mathcal{H}_{\sigma}^{n}
$$

for bosons and

$$
\mathcal{F}_{\tau}=\bigoplus_{n=0}^{\infty} \mathcal{H}_{\tau}^{n}
$$

for fermions. Once each Fock-space is constructed, the usual procedure runs as follows. Let $\psi(\mathbf{r})$ and its hermitian conjugate $\psi(\mathbf{r})^{\dagger}$ be operators acting on the Fock-space and satisfying:

$$
\begin{gathered}
{\left[\psi(\mathbf{r}), \psi\left(\mathbf{r}^{\prime}\right)\right]_{\mp}=0} \\
{\left[\psi(\mathbf{r})^{\dagger}, \psi\left(\mathbf{r}^{\prime}\right)^{\dagger}\right]_{\mp}=0} \\
{\left[\psi(\mathbf{r}), \psi\left(\mathbf{r}^{\prime}\right)^{\dagger}\right]_{\mp}=\delta_{\mathbf{r}-\mathbf{r}^{\prime}}}
\end{gathered}
$$

where $\delta\left(\mathbf{r}-\mathbf{r}^{\prime}\right)$ is the Dirac delta function. For any operators $A$ and $B$, the brackets are defined by:

$$
[A, B]_{\mp} \stackrel{\text { def }}{=} A B \mp B A
$$


Corresponding to the $n$ particle wave function $\Psi_{n}\left(\mathbf{r}_{1}, \ldots, \mathbf{r}_{n}\right)$ of the standard formulation, the $n$ particle state in the Fock-space is defined:

$$
\left|\psi_{n}\right\rangle \stackrel{\text { def }}{=}(n !)^{-\frac{1}{2}} \int d^{3} r_{1} \cdots \int d^{3} r_{n} \psi\left(\mathbf{r}_{1}\right)^{\dagger} \cdots \psi\left(\mathbf{r}_{n}\right)^{\dagger}|0\rangle \Psi_{n}\left(\mathbf{r}_{1}, \ldots, \mathbf{r}_{n}\right)
$$

which can be shown to be an eigenvector (with eigenvalue $n$ ) of the particle number operator:

$$
N \stackrel{\text { def }}{=} \int d^{3} r \psi(\mathbf{r})^{\dagger} \psi(\mathbf{r})
$$

The connection between the two representations is given by:

$$
\Psi_{n}\left(\mathbf{r}_{1}, \cdots, \mathbf{r}_{n}\right)=(n !)^{-\frac{1}{2}}\left\langle 0\left|\psi\left(\mathbf{r}_{1}\right) \cdots \psi\left(\mathbf{r}_{n}\right)\right| \Psi_{n}\right\rangle
$$

In general, an arbitrary vector of the Fock-space:

$$
|\Psi\rangle=\sum_{n=0}^{\infty}\left|\Psi_{n}\right\rangle
$$

will not be an eigenstate of the particle number operator.

Corresponding to the kinetic energy operator of standard wave mechanics, an operator in the Fock-space is defined as:

$$
T \stackrel{\text { def }}{=} \int d^{3} r \psi^{\dagger}(\mathbf{r}) T_{1}(r) \psi(\mathbf{r})
$$

and it is easy to see that:

$$
T^{\dagger}=T
$$

It can also be shown that:

$T\left|\Psi_{n}\right\rangle=(n !)^{-\frac{1}{2}} \int d^{3} r_{1} \cdots \int d^{3} r_{n} \Psi^{\dagger}\left(\mathbf{r}_{1}\right) \cdots \Psi^{\dagger}\left(\mathbf{r}_{n}\right)|0\rangle \sum_{i=1}^{n} T_{1}\left(\mathbf{r}_{i}\right) \Psi_{n}\left(\mathbf{r}_{1}, \cdots, \mathbf{r}_{n}\right)$

Analogously, if there is a pairwise interaction potential $V_{2}\left(\mathbf{r}, \mathbf{r}^{\prime}\right)$, the operator:

$$
V \stackrel{\text { def }}{=} \frac{1}{2} \int d^{3} r \int d^{3} r^{\prime} \psi^{\dagger}(\mathbf{r}) \psi^{\dagger}\left(\mathbf{r}^{\prime}\right) V_{2}\left(\mathbf{r}, \mathbf{r}^{\prime}\right) \psi\left(\mathbf{r}^{\prime}\right) \psi(\mathbf{r})
$$

is defined on the Fock-space. Its action on $\left|\Psi_{n}\right\rangle$ is given by:

$$
V\left|\Psi_{n}\right\rangle=(n !)^{-\frac{1}{2}} \int d^{3} r_{1} \cdots \int d^{3} r_{1}\left[V, \psi^{\dagger}\left(\mathbf{r}_{n}\right) \cdots \psi^{\dagger}\left(\mathbf{r}_{1}\right)\right]|\mathbf{0}\rangle \Psi_{n}\left(\mathbf{r}_{1} \ldots \mathbf{r}_{n}\right)
$$


and it follows that:

$$
\begin{aligned}
V\left|\Psi_{n}\right\rangle & =(n !)^{-\frac{1}{2}} \int d^{3} r_{1} \cdots \int d^{3} r_{1} \psi^{\dagger}\left(\mathbf{r}_{n}\right) \cdots \psi^{\dagger}\left(\mathbf{r}_{1}\right)|\mathbf{0}\rangle \\
& \times \sum_{i=1}^{n} \sum_{j=1}^{i-1} V_{2}\left(\mathbf{r}_{i}, \mathbf{r}_{j}\right) \Psi_{n}\left(\mathbf{r}_{1}, \ldots, \mathbf{r}_{n}\right)
\end{aligned}
$$

It can be shown that that the following equations holds:

$$
\begin{aligned}
& T_{n} \Psi_{n}\left(\mathbf{r}_{1}, \cdots, \mathbf{r}_{n}\right)=(n !)^{-\frac{1}{2}}\left\langle 0\left|\Psi\left(\mathbf{r}_{1}\right) \cdots \Psi\left(\mathbf{r}_{n}\right) T\right| \Psi_{n}\right\rangle \\
& V_{n} \Psi_{n}\left(\mathbf{r}_{1}, \cdots, \mathbf{r}_{n}\right)=(n !)^{-\frac{1}{2}}\left\langle 0\left|\Psi\left(\mathbf{r}_{1}\right) \cdots \Psi\left(\mathbf{r}_{n}\right) V\right| \Psi_{n}\right\rangle
\end{aligned}
$$

where:

$$
\begin{gathered}
T_{n} \stackrel{\text { def }}{=} \sum_{i=1}^{n} T_{1}\left(\mathbf{r}_{i}\right) \\
V_{n} \stackrel{\text { def }}{=} \sum_{i>j=1}^{n} V_{2}\left(\mathbf{r}_{i}, \mathbf{r}_{j}\right)
\end{gathered}
$$

The equivalence with wave mechanics can now be established as follows. If $\Psi_{n}\left(\mathbf{r}_{1}, \cdots, \mathbf{r}_{n}\right)$ satisfies the $n$ particle Schrödinger wave equation with Hamiltonian (21), it follows that in the Fock-space formulation $\left|\Psi_{n}\right\rangle$ must satisfy the Fock-space Schrödinger equation:

$$
\left[i \hbar\left(\frac{\partial}{\partial t}\right)-H\right]\left|\Psi_{n}\right\rangle=0
$$

with $H \stackrel{\text { def }}{=} T+V$ : given by:

$$
\begin{aligned}
H & =\int d^{3} r \psi^{\dagger}(\mathbf{r})\left[T_{1}+V_{1}(\mathbf{r})\right] \psi(\mathbf{r}) \\
& +\frac{1}{2} \int d^{3} r \int d^{3} r^{\prime} \psi^{\dagger}(\mathbf{r}) \psi^{\dagger}\left(\mathbf{r}^{\prime}\right) V_{2}\left(\mathbf{r}, \mathbf{r}^{\prime}\right) \psi\left(\mathbf{r}^{\prime}\right) \psi(\mathbf{r})
\end{aligned}
$$

It is important to remark that the $n$ particle Schrödinger wave equation is not completely equivalent to its analogue in the Fock-space formalism. Only solutions of the Fock-space equation which are eigenvectors of the particle number operator with particle number $n$ can be solutions of the corresponding $n$ particle Schrödinger wave equation. On the other hand, not all the solutions of the $n$ particle Schrödinger wave equation can be solutions of the Fock equation, only those which are adequately symmetrized do. So, both conditions, defined particle number and symmetrization, must hold in order that both formalisms are equivalent. 


\subsection{Creation and annihilation operators}

The standard manner to handle with the equations in Fock-space is to write physical magnitudes in terms of creation and annihilation operators. To do so, one makes the following expansion:

$$
\psi(\mathbf{r})=\sum_{k} a_{k} u_{k}(\mathbf{r})
$$

The coefficients of the expansion are the annihilation operators:

$$
a_{k} \stackrel{\text { def }}{=} \int d^{3} r u_{k}^{*}(\mathbf{r}) \psi(\mathbf{r})
$$

A similar expansion stands for the creation operator: $a_{k}^{\dagger}$. In quantum field theory, it is commonly assumed that the action of the operator $a_{k}^{\dagger}$ describes the "creation of a particle" with wave function $u_{k}(\mathbf{r})$. In a similar way, is interpreted that the action of $a_{k}$ describes the "annihilation of a particle". It can be shown, that these operators satisfy the commutation relations:

$$
\begin{gathered}
{\left[a_{k}, a_{l}\right]_{\mp}=0} \\
{\left[a_{k}^{\dagger}, a_{l}^{\dagger}\right]_{\mp}=0} \\
{\left[a_{k}, a_{l}^{\dagger}\right]_{\mp}=\delta_{k l}} \\
N_{k} \stackrel{\text { def }}{=} a_{k}^{\dagger} a_{k}
\end{gathered}
$$

We can cast these last equations in a more familiar form, using the "[, ]" symbol for bosonic commutation relations and the " $\{$,$\} " symbol for fermionic$ (anti)commutation relations. Then for bosons we have:

$$
\begin{gathered}
{\left[a_{\alpha} ; a_{\beta}^{\dagger}\right]=a_{\alpha} a_{\beta}^{\dagger}-a_{\beta}^{\dagger} a_{\alpha}=\delta_{\alpha \beta} I} \\
{\left[a_{\alpha}^{\dagger} ; a_{\beta}^{\dagger}\right]=0} \\
{\left[a_{\alpha} ; a_{\beta}\right]=0}
\end{gathered}
$$

and for fermions, (with $C_{\alpha}^{\dagger}$ and $C_{\alpha}$ playing the role of fermionic creation and annihilation operators respectively) we have:

$$
\begin{gathered}
\left\{C_{\alpha} ; C_{\beta}^{\dagger}\right\}=C_{\alpha} C_{\beta}^{\dagger}+C_{\beta}^{\dagger} C_{\alpha}=\delta_{\alpha \beta} I \\
\left\{C_{\alpha}^{\dagger} ; C_{\beta}^{\dagger}\right\}=0
\end{gathered}
$$




$$
\left\{C_{\alpha} ; C_{\beta}\right\}=0
$$

Substitution of (50) in (49) yields:

$$
H=\sum_{k l} a_{k}^{\dagger} T_{k l} a_{l}+\frac{1}{2} \sum_{k l p q} a_{k}^{\dagger} a_{l}^{\dagger} V_{k l p q} a_{p} a_{q}
$$

where the matrix elements $T_{k l}$ and $V_{k l p q}$ are given by:

$$
\begin{gathered}
T_{k l}=\int d^{3} r u_{k}^{*}(\mathbf{r})\left[\left(-\frac{\hbar^{2} \nabla^{2}}{2 m}\right)+V_{1}(\mathbf{r})\right] u_{l}(\mathbf{r}) \\
V_{k l p q}=\int d^{3} r \int d^{3} r^{\prime} u_{k}^{*}(\mathbf{r}) u_{l}^{*}\left(\mathbf{r}^{\prime}\right) V_{2}\left(\mathbf{r}, \mathbf{r}^{\prime}\right) u_{p}\left(\mathbf{r}^{\prime}\right) u_{q}(\mathbf{r})
\end{gathered}
$$

and similar expressions can be found for more general obserables.

\subsection{Using the $\mathfrak{Q}$-space}

We have constructed two spaces whose vectors make no reference to particle indexation and, besides, particles are not labeled in any step of the formal construction. This is possible because these spaces are constructed using the non classical part of $\mathfrak{Q}$, which may refer to intrinsically indistinguishable entities. Vectors in these spaces are only distinguished by the occupation number in each (energy) level. With these tools and using the language of $\mathfrak{Q}$, the formalism of quantum mechanics may be completely rewritten giving a straightforward answer to the problem of giving a formulation of quantum mechanics in which intrinsical indistinguishability is taken into account from the beginning, without artificially introducing extra postulates.

Let us first show that the $\mathfrak{Q}$-space is useful to provide a states space analogous to Fock-space. With this aim, we make the following association in order to turn the notation similar to that of standard quantum mechanics. For each quasi-function $f_{\epsilon_{i_{1}} \epsilon_{i_{2}} \ldots \epsilon_{i_{n}}}$ of the quasi-sets $\mathcal{F}$ or $\mathcal{O} \mathcal{F}$ constructed above, we will write:

$$
\left.\alpha f_{\epsilon_{i_{1}} \epsilon_{i_{2}} \ldots \epsilon_{i_{n}}} \stackrel{\text { def }}{=} \alpha \mid \epsilon_{i_{1}} \epsilon_{i_{2}} \ldots \epsilon_{i_{n}}\right)
$$

with the obvious corresponding generalization for linear combinations.

Let us recall again that in $\left.\mid \epsilon_{i_{1}} \epsilon_{i_{2}} \ldots \epsilon_{i_{n}}\right) \in \mathcal{F}$, the order of the indexes has no meaning. But in $\left.\mid \epsilon_{i_{1}} \epsilon_{i_{2}} \ldots \epsilon_{i_{n}}\right) \in \mathcal{O} \mathcal{F}$, the order makes sense.

As we have already pointed out that, to avoid particle labeling in the expressions for observables, no reference to particle indexation should appear in their corresponding operators. For that reason we will only use creation 
and annihilation operators. To do so, we construct creation and annihilation acting on $\mathfrak{Q}$-spaces. In the rest of this section, we will develop the idea of the symmetrized products (15) and (17) discussed in 3.2. We will first develop the construction for bosons and later fermions. We will use creation and annihilation operators and instead of postulating commutation relations, we will deduce them from their definitions and the properties of the vectors of the $\mathfrak{Q}$-spaces (following an analogous procedure as that exposed, for example, in [1, Chap. 17]).

\subsection{Bosonic states}

For bosons, the procedure is similar to the procedure of the standard approach for, as we have remarked earlier, a scalar product naturally arises from the symmetric product (15). This implies that, once normalized to unity, the vectors $\mid \alpha \beta \gamma \ldots)$ constructed using $\mathfrak{Q}$, are equivalent to the symmetrized vectors $|\alpha \beta \gamma \ldots\rangle$ for bosonic states. This is so, because permutations alter the vector in none of the spaces.

Suppose then that vectors $\mid \alpha \beta \gamma \ldots)$ are normalized to unity. If $\zeta$ represents an arbitrary collection of indexes, we define:

$$
\left.\left.a_{\alpha}^{\dagger} \mid \zeta\right) \propto \mid \alpha \zeta\right)
$$

in such a way that the proportionality constant satisfies

$$
\left.\left.a_{\alpha}^{\dagger} a_{\alpha} \mid \zeta\right)=n_{\alpha} \mid \zeta\right)
$$

Then, it follows that:

$$
\left(\left(\zeta \mid a_{\alpha}^{\dagger}\right)\left(a_{\alpha} \mid \zeta\right)\right)=n_{\alpha}
$$

obtaining the usual

\section{Definition 4.1}

$$
\left.\left.a_{\alpha} \mid \ldots n_{\alpha} \ldots\right)=\sqrt{n_{\alpha}} \mid \ldots n_{\alpha}-1 \ldots\right)
$$

On the other hand,

$$
\left.\left.a_{\alpha} a_{\alpha}^{\dagger} \mid \ldots n_{\alpha} \ldots\right)=K \sqrt{n_{\alpha}+1} \mid \ldots n_{\alpha} \ldots\right)
$$

where $K$ is a proportionality constant. If we apply $a_{\alpha}^{\dagger}$ once again:

$$
\left.\left.a_{\alpha}^{\dagger} a_{\alpha} a_{\alpha}^{\dagger} \mid \ldots n_{\alpha} \ldots\right)=K^{2} \sqrt{n_{\alpha}+1} \mid \ldots n_{\alpha}+1 \ldots\right)
$$

and using (63):

$$
\left.\left.\left(a_{\alpha}^{\dagger} a_{\alpha}\right) a_{\alpha}^{\dagger} \mid \ldots n_{\alpha} \ldots\right)=\sqrt{n_{\alpha}+1} K \mid \ldots n_{\alpha}+1 \ldots\right)
$$

so $K=\sqrt{n_{\alpha}+1}$. Then, 


\section{Definition 4.2}

$$
\left.\left.a_{\alpha}^{\dagger} \mid \ldots n_{\alpha} \ldots\right)=\sqrt{n_{\alpha}+1} \mid \ldots n_{\alpha}+1 \ldots\right)
$$

Once this is established, let us obtain the commutation relations. By a straightforward computation, we see that:

$$
\left.\left.\left(a_{\alpha} a_{\beta}^{\dagger}-a_{\beta}^{\dagger} a_{\alpha}\right) \mid \psi\right)=\delta_{\alpha \beta} \mid \psi\right)
$$

which is the same as:

$$
\left[a_{\alpha} ; a_{\beta}^{\dagger}\right]=\delta_{\alpha \beta} I
$$

In an analogous way we can show that:

$$
\left[a_{\alpha} ; a_{\beta}\right]=\left[a_{\alpha}^{\dagger} ; a_{\beta}^{\dagger}\right]=0
$$

This shows that the (bosonic) commutation relations that are obtained in $\mathfrak{Q}$-space are the same ones as in the standard Fock-space.

\subsection{Fermionic states}

For the fermionic case, we will use $C_{0}$ equipped with the antisymmetric product given by equation (17). We define the creator operator $C_{\alpha}^{\dagger}$ as follows:

Definition 4.3 Let $\zeta$ represent a collection of indexes with non null occupation number, then

$$
\left.\left.C_{\alpha}^{\dagger} \mid \zeta\right)=\mid \alpha \zeta\right)
$$

Note that if $\alpha$ was already in the collection $\zeta$, then $\mid \alpha \zeta)$ is a vector with null norm. To have null norm implies that $(\psi \mid \alpha \zeta)=0$ for all $\mid \psi)$. Then, if a given vector has null norm, its scalar product with any other vector in the space is zero. It follows that in the case that a system were eventually in a state of null norm, the probability of observing it would be zero. In the same way, if a linear combination of null norm vectors were added to the vector representing the state of a system, this addition would not give place to observable results. It follows then that null norm vectors do not represent real physical states, and the same holds for linear combinations of them. Moreover, adding a vector of null norm to any other one does not produce observable affects, because the terms of null norm do not contribute to the mean values or to the probabilities. In order to express this situation, we define the following relation: 
Definition 4.4 Two vectors $\mid \varphi$ ) and $\mid \psi$ ) are similar (and we will write $(\varphi) \cong(\psi)))$ if the difference between them is a linear combination of null norm vectors.

Let us now compute the effect of applying $C_{\alpha}$ to the vectors of $\mathcal{O F}$. Using Definition (4.3) we find that:

$$
\left(\zeta \mid C_{\alpha}=(\alpha \zeta \mid\right.
$$

Then, for any vector $\mid \psi)$ :

$$
\left(\zeta\left|C_{\alpha}\right| \psi\right)=(\alpha \zeta \mid \psi)=0
$$

for $\alpha \in \zeta$ or $(\psi \mid \alpha \zeta)=0$. Then, if we choose $\mid \psi)=\mid 0)$ it follows that:

$$
\left(\zeta\left|C_{\alpha}\right| 0\right)=(\alpha \zeta \mid 0)=0
$$

and thus we obtain that $\left.C_{\alpha} \mid 0\right)$ is orthogonal to any vector which contains $\alpha$ and to any vector which does not contain $\alpha$. Then, it is orthogonal to any vector, and for that reason, it has to be a linear combination of null norm vectors. Then, we do not loose anything if we establish $\left.C_{\alpha} \mid 0\right)=\overrightarrow{0}$. In an analogous way we can assert that:

$$
\left.C_{\alpha} \mid(\sim \alpha) \cdots\right)=\overrightarrow{0}
$$

where $(\sim \alpha)$ means that $\alpha$ has occupation number zero, and the dots mean that the other levels have arbitrary occupation numbers. Using (4.4) we can also write:

$$
\left.C_{\alpha} \mid 0\right) \cong \overrightarrow{0}
$$

and

$$
\left.C_{\alpha} \mid(\sim \alpha) \ldots\right) \cong \overrightarrow{0}
$$

In what follows we will use $\cong$ when it be necessary, but the same results are obtained if we replace $\cong$ by the extensional equality. Making $|\psi|=\mid \alpha$ ) in (75) it follows that:

$$
\left(\zeta\left|C_{\alpha}\right| \alpha\right)=(\alpha \zeta \mid \alpha)=0
$$

in any case except for $\mid \zeta)=\mid 0)$. In that case, $\left(0\left|C_{\alpha}\right| \alpha\right)=1$. Then, it follows that $\left.\left.C_{\alpha} \mid \alpha\right) \cong \mid 0\right)$. In an analogous way we obtain:

$$
\left.\left.C_{\alpha} \mid \alpha \zeta\right) \cong \mid(\sim \alpha) \zeta\right)
$$


if $\alpha$ does not belongs to $\zeta$. But in the case that $\alpha$ belongs to $\zeta$, (i.e., the occupation number is greater than 1) we have that $\mid \alpha \zeta)$ has null norm, and so:

$$
\left.\left(\alpha \zeta\left|C_{\alpha}^{\dagger}\right| \psi\right)=(\alpha \zeta \mid \alpha \psi)=0, \forall \mid \psi\right)
$$

From this equation it follows that:

$$
\left.\left(\psi\left|C_{\alpha}\right| \alpha \zeta\right)=0, \forall \mid \psi\right)
$$

and so, $\left.C_{\alpha} \mid \alpha \zeta\right)$ has null norm too.

Now, let us find the anti-commutation relations obeyed by the fermionic creation and annihilation operators. Let us first calculate the commutation relation between $C_{\alpha}$ and $C_{\beta}^{\dagger}$. To do so, let us first study the relationship between $\mid \alpha \beta) \in \mathcal{O} \mathcal{F}$ and $\mid \beta \alpha) \in \mathcal{O} \mathcal{F}$. With this aim, consider the vector $\mid \alpha \beta)+\mid \beta \alpha)$ and perform the product of this sum with another arbitrary vector. It suffices to study what happens with basis vectors. The product yields trivially zero for any vector different from $\mid \alpha \beta)$ or $\mid \beta \alpha)$. Making the product with $\mid \alpha \beta)$ we obtain:

$$
\begin{gathered}
(\alpha \beta|[\mid \alpha \beta)+| \beta \alpha)]=(\alpha \beta|| \alpha \beta)+(\alpha \beta|| \beta \alpha)= \\
\delta_{\alpha \alpha} \delta_{\beta \beta}-\delta_{\alpha \beta} \delta_{\beta \alpha}+\delta_{\alpha \beta} \delta_{\alpha \alpha}-\delta_{\alpha \alpha} \delta_{\beta \beta}=1-0+0-1=0
\end{gathered}
$$

The same conclusion holds if we multiply it by $\mid \beta \alpha)$. Then, it follows that $\mid \alpha \beta)+\mid \beta \alpha$ ) is a linear combination of null norm vectors (which we will denote by $\mid n n l c))$ and, thus:

$$
\mid \alpha \beta)=-\mid \beta \alpha)+\mid n n l c)
$$

We do not care about which is the particular null norm linear combination, because it has no observable effects. Now, we can calculate

$$
\left.\left.\left.\left.C_{\alpha}^{\dagger} C_{\beta}^{\dagger}|\psi\rangle=|\alpha \beta| \psi\right)={ }_{-} \mid \beta \alpha \psi\right)+\mid n n l c\right)=-C_{\beta}^{\dagger} C_{\alpha}^{\dagger}|\psi\rangle+\mid n n l c\right)
$$

and thus

$$
\left.\left.\left\{C_{\alpha}^{\dagger} ; C_{\beta}^{\dagger}\right\} \mid \psi\right)=\mid n n l c\right)
$$

Then, we do not loose generality if we set

$$
\left.\left\{C_{\alpha}^{\dagger} ; C_{\beta}^{\dagger}\right\} \mid \psi\right)=0
$$

In an analogous way, we conclude that

$$
\left.\left\{C_{\alpha} ; C_{\beta}\right\} \mid \psi\right)=0
$$


Now let us calculate the commutation relation between $C_{\alpha}$ and $C_{\beta}^{\dagger}$. Suppose first that $\alpha \neq \beta$. If $\alpha \notin \psi$ or $\beta \in \psi$ then it is clear that

$$
\left.\left\{C_{\alpha} ; C_{\beta}^{\dagger}\right\} \mid \psi\right) \approx \overrightarrow{0}
$$

If $\alpha \in \psi$ and $\beta \notin \psi$, suppose (without loss of generality), that $\alpha$ is the first symbol in the list of $\psi$. Then,

$$
\begin{gathered}
\left.\left.\left.\left\{C_{\alpha} ; C_{\beta}^{\dagger}\right\} \mid \psi\right)=C_{\alpha} \mid \beta \psi\right)+C_{\beta}^{\dagger} \mid \psi(\sim \alpha)\right) \cong \\
\cong-\mid \beta \psi(\sim \alpha))+\mid \beta \psi(\sim \alpha))=\overrightarrow{0}
\end{gathered}
$$

If $\alpha=\beta$, and $\alpha \in \psi$, then

$$
\begin{aligned}
\left.\left\{C_{\alpha} ; C_{\alpha}^{\dagger}\right\} \mid \psi\right) & \left.\left.=C_{\alpha} \mid \alpha \psi\right)+C_{\alpha}^{\dagger} \mid \psi(\sim \alpha)\right) \cong \\
& \cong \overrightarrow{0}+|\psi|=|\psi|
\end{aligned}
$$

If $\alpha=\beta$, and $\alpha \notin \psi$, then

$$
\begin{aligned}
\left.\left\{C_{\alpha} ; C_{\alpha}^{\dagger}\right\} \mid \psi\right) & \left.\left.=C_{\alpha} \mid \alpha \psi\right)+C_{\alpha}^{\dagger} \mid \psi(\sim \alpha)\right) \cong \\
& \cong|\psi|+\overrightarrow{0}=|\psi|
\end{aligned}
$$

So, in any case, we recover the relation

$$
\left.\left.\left\{C_{\alpha} ; C_{\alpha}^{\dagger}\right\} \mid \psi\right) \cong \delta_{\alpha \beta} \mid \psi\right)
$$

and then, we can set

$$
\left\{C_{\alpha} ; C_{\alpha}^{\dagger}\right\}=\delta_{\alpha \beta} .
$$

Thus, we have shown that the same commutation relations hold, as the standard formalism hold in $\mathfrak{Q}$-space. This means that both formulations are equivalent, for all the interesting information is contained in the commutation relations. In the following section, we discuss some features of this new formulation.

\section{Discussion}

We have shown that it is possible to construct the quantum mechanical formalism for indistinguishable particles without labeling them in any step. To do so, we have built a vector space with inner product, the $\mathfrak{Q}$-space, using the non-classical part of $\mathfrak{Q}$, the generalization of ZFU, to deal with indistinguishable elements. Vectors in $\mathfrak{Q}$-space refer only to occupation numbers and permutations operators act as the identity operator, reflecting in the 
formalism the fact of unobservability of permutations, already expressed in terms of the formalism of $\mathfrak{Q}$.

We have also argued that it is useful to represent operators (which are intended to represent observable quantities) as combinations of creator and annihilation operators, in order to avoid particle indexation in the expression of observable quantities. We have shown that creation and annihilation operators which act on $\mathfrak{Q}$-space can be constructed. We have proved that they obey the usual commutation and anticommutation relations for bosons and fermions respectively, and this means that our construction is equivalent to that of the Fock-space formulation of quantum mechanics. Thus, using the results reviewed in section 4 , this implies that we can recover the $n$-particles wave equation using $\mathfrak{Q}$-space in the same way as in the standard theory. Though both formulations are equivalent 'for all practical purposes', when subjected to careful analysis, the conceptual difference turns very important. Our construction avoids the LTPSF by constructing the state spaces using $\mathfrak{Q}$, a theory which can deal with truly indistinguishable entities, and so, it gives an alternative (and radical) answer to the problems posed in [26], so as (we guess) answers Manin's problem posed in [21].

This last point seems remarkable, for our construction incorporates intrinsical indistinguishability from the beginning. Thus, our approach fulfills not only Post's claim already mentioned, but also both Manin's claim that we should find an adequate "set theory" for expressing collections of indistinguishable quanta, and Heisenberg's idea that new tools (perhaps new logical tools) seem to be justified in approaching "new fields of experience", as we see in the quotation at the beginning of our Introduction (which we invite the reader to have a new look), so as also the following quotation from Manin:

"In accordance with Hilbert's prophecy, we are living in Cantor's paradise. So we are bound to be tempted. ...

"We should consider the possibilities of developing a totally new language to speak about infinity. ${ }^{5}$ Classical critics of Cantor (Brouwer et al.) argued that, say, the general choice axiom is an illicit extrapolation of the finite case.

"I would like to point out that this is rather an extrapolation of common-place physics, where we can distinguish things, count them, put them in some order, etc. New quantum physics has shown us models of entities with quite different behavior. Even

\footnotetext{
${ }^{5}$ Set theory is also known as the theory of the 'infinite'.
} 
'sets' of photons in a looking-glass box, or of electrons in a nickel piece are much less Cantorian than the 'set' of grains of sand. In general, a highly probabilistic 'physical infinity' looks considerably more complicated and interesting than a plain infinity of 'things'."

Manin is right. The foundation analysis of a living science is of course a difficult problem. As far as we go to the details, science itself changes, and in certain sense axiomatization becomes (as it was considered long time ago)

just a cosmetics to the scientific theories. But this conclusion does not make justice to the advance of the modern techniques of logic and mathematics. A careful look to the foundational details may illuminate conceptual problems, open new windows and show new mathematical and logical questions. Once more referring to Yuri Manin, we think he is right in saying that "The twentieth century return to Middle Age scholastics taught us a lot about formalisms. Probably it is time to look outside again. Meaning is what really matters" [21]. Quantum physics is a wonderful land to look at. As our author also says,

"The development of the foundations of physics in the twentieth century has taught us a serious lesson. Creating and understanding these foundations turned out to have very little to do with the epistemological abstractions which were of such importance to the twentieth century critics of the foundations of mathematics: finiteness, consistency, constructibility, and, in general, the Cartesian notion of intuitive clarity. Instead, completely unforeseen principles moved into the spotlight: complementarity, and the nonclassical, probabilistic truth function. The electron is infinite, capricious, and free, and does not at all share our love for algorithms." [22, pp. 82-83]

\section{References}

[1] Ballentine, L. E.: 2000, Quantum Mechanics: A Modern Development, Singapore, World Scientific.

[2] Becker, J.: 2008, Topics on Quasi-Set Theory and on its Applications to the Philosophy of Quantum Physics (in Portuguese), MSc dissertation, Federal University of Santa Catarina. 
[3] Bokulich, A.: 2006, "Heisenberg Meets Kuhn: Closed Theories and Paradigms", Philosophy of Science, 73, 90107.

[4] da Costa, N. C. A. and Krause, D.: 1994, "Schrödinger logics", Studia Logica 53 (4), 533-550.

[5] da Costa, N. C. A. and Krause, D.: 1997, "An intensional Schrödinger logic", Notre Dame Journal of Formal Logic 38 (2), 179-194.

[6] da Costa, N. C. A. and Krause, D.: 2007, "Logical and Philosophical Remarks on Quasi-Set Theory", Logic Journal of the Interest Group in Pure and Applied Logics, 15, 1-20.

[7] Dalla Chiara, M. L. and Toraldo di Francia, G.: 1995, "Identity Questions from Quantum Theory", in Gavroglu, K. et. al., (eds.), Physics, Philosophy and the Scientific Community, Dordrecht, Kluwer Academic Publishers, 39-46.

[8] Dalla Chiara, M. L., Giuntini, R. and Krause, D.: 1998, "Quasiset Theories for Microobjects: A Comparision", in Castellani, E. (ed.), Interpreting bodies: Classical and quantum objects in modern physics, Princeton University Press, Princeton.

[9] Domenech, G. and Holik, F.: 2007, "A discussion on particle number and quantum indistinguishability", Found. Phys. 37 (6), 855-878.

[10] Falkenburg, B.: 2007, Particle Metaphysics: A Critical Account of Subatomic Reality, Springer

[11] French, S. and Krause, D.: 2003, "Quantum Vagueness", Erkenntnis $59,97-124$.

[12] French, S. and Krause, D.: 2006, Identity in Physics: A Historical, Philosophical, and Formal Analysis, Oxford, Oxford University Press.

[13] Halmos P.: 1963, Naive Set Theory, D. Van Nostrand Company.

[14] Halvorson, H. P. and Clifton, R.: 2001, "Are Rindler quanta real? Inequivalent particle concepts in quantum field theory", British Journal for the Philosophy of Science, 52, 417-470.

[15] Heisenberg, W.: 1979, "Recent Changes in the Foundations of Exact Science", in F. C. Hayes (trans.), Philosophical Problems of Quantum Physics, Woodbridge, CT: Ox Bow Press, 1126. 
[16] Krause, D.: 1992, "On a quasi set theory", Notre Dame J. of Formal Logic 33, 402-411.

[17] Krause, D.: 2003, "Why quasi-sets?", Boletim da Sociedade Paranaense de Matemática 20, 73-92.

[18] Krause, D., Sant'Anna, A. S. and Volkov, A. G.: 1999, "Quasi-set theory for bosons and fermions: quantum distributions", Foundations of Physics Letters 12 (1), pp. 51-66.

[19] Krause, D., Sant'Anna, A. S. and Sartorelli, A.: 2005, "A critical study on the concept of identity in Zermelo-Fraenkel like axioms and its relationship with quantum statistics", Logique \& Analyse, 189-192, 231-260.

[20] Leibinz, G. W.: 1995, Philosophical Writings, Ed. by G. H. R. Parkinson, London, Everyman.

[21] Manin, Yu. I.: 1976, "Mathematical Problems I: Foundations", in Browder, F. E. (ed.): 1976, Mathematical Problems Arising from Hilbert Problems, Proceedings of Symposia in Pure Mathematics, Vol. XXVIII, Providence, American Mathematical Society, 36.

[22] Manin, Yu. I.: 1977, A course in mathematical logic, Springer-Verlag.

[23] Mittelstaedt, P.: 1998, The interpretation of quantum mechanics and the measurement process, Cambridge University Press.

[24] Post, H.: 1963, "Individuality and physics", The Listener, 10 October, 534-537, reprinted in Vedanta for East and West 132, 1973, 14-22.

[25] Redhead, M. and Teller, P.: 1991, "Particles, particle labels, and quanta: the toll of unacknowledged metaphysics", Foundations of Physics 21, pp. 43-62.

[26] Redhead, M. and Teller, P.: 1992, "Particle labels and the theory of indistinguishable particles in quantum mechanics", British Journal for the Philosophy of Science 43, pp. 201-218. pp. 14-22.

[27] Robertson, B.: 1973, "Introduction to field operators in quantum mechanics", Amer. J. Phys. 41 (5), 678-690

[28] Penrose, R.: 1989 The emperors new mind, Oxford, Oxford Un. Press.

[29] Sant'Anna, A.: 2005, "Labels for non individuals?", Found. Phys. Lett. 18, 0894-9875. 
[30] Schrödinger, E.: 1952, Science and Humanism, Cambridge Un. Press, Cambridge.

[31] Schrödinger, E.: 1998, "What is an elementary particle?", reprinted in Castellani, E. (ed.), Interpreting bodies: classical and quantum objects in modern physics, Princeton Un. Press, 197-210.

[32] Teller, P.: 1995, An Interpretative Introduction to Quantum Field Theory, Princeton, Princeton University Press.

[33] Toraldo di Francia, G.: 1981, The Investigation of the Physical World, Cambridge Un. Press.

[34] Weyl, H.: 1949, Philosophy of mathematics and natural science, Princeton, Princeton University Press. 